tung der Kammer aus dem System und der Abnahme und Wiederaufsetzung der Deckel, nur ungefähr 10 Minuten. Der von den Elektroden abfallende Staub wird von einem Förderwerk einer gemeinsamen, an den neun Kammern vorbeiführenden Förderschnecke zugeleitet, die sich in Wagen entleert.

Filterproben haben gezeigt, daB unter günstigen Arbeitsverhältnissen die Anlage $80-90 \%$ der in dem Gas schwebenden Stoffe niederschlägt. Für die ganze Zeit, wälrend welcher sie in Tätigkeit gewesen ist, hat sich der Durchschnitt etwas. niedriger gestellt. Im vergangenen Frühjahr wurden 6-8 t in 24 Stunden niedergeschlagen. Die Menge der zu behandelnden Gase hetrug zu jener Zeit 5660 bis $8495 \mathrm{cbm}$ in der Minute; sie traten bei $100-150^{\circ}$ in die Fällkammer ein.

Eine der größten Schwierigkeiten dieser Anlage bestand darin, die feinen Asbestfasern und Glimmerteilchen der Entladungselektroden leitfähig zu erhalten. Bei gewöhnlicher Zimmertemperatur nehmen sie genügend Feuchtigkeit aus der Luft auf und auch bei höheren Temperaturen, wenn die Gase Spuren von Schwefelsäure oder anderen leitfähigen Stoffen enthalten. Den hier zu behandelnden Gasen werden aber bisweilen durch ihren hohen und wechselnden Zinkoxydgehalt alle leitfähigen Stoffe entzogen, wodurch die Leitfähigkeit und Wirksamkeit der Elektrodenfasern stark beeinträchtigt wird. An der Beseitigung dieses Ubelstandes wurde im vergangenen Frühjahre gearbeitet.

Die Balaklalahütte hat im letzten Sommer den Betrieb bis auf weiteres einstellen müssen, da sie der oben erwähnten gerichtsseitigen Verfügung, nämlich sämtliche festen Stoffe aus dem Hüttenrauch abzuscheiden, den Gehalt von Schwefeldioxyd nie über $0,75 \%$ steigen zu lassen und keinerlei Schaden $z u$ verursachen, nicht buchstäblich hat nach. kommen können. Die Hauptschuld trifft das Schwefeldioxyd. Die cot t r ell sche Anlage hat sich dagegen durchaus bewährt. Sie hat ungefähr 135000 Doll. gekostet.

Eine weitere Verwertung hat das Verfahren in den Riverside Portland Cement Works in Riverside im südlichen Californien gefundèn, um den Staub aus den Ofengasen niederzuschlagen, der auf die benachbarten Orangengärten sehr schädlich wirkte. Die Anlage, die 100000 Doll. gekostet hat, arbeitet zur Zufriedenheit und liefert als Nebenprodukt aus dem niedergeschlagenen Staub Kalidünger.

Auch zum Ausbringen von $B \mathrm{l}$ e i a u $\mathrm{s}$ u p . ferkonvertergasen wird das Verfahren neuerdings angewendet. Zum Auffangen des Staubes werden dabei keine ebenen Platten benutzt, sondern Röhren, in deren Mitte sich die Entladungselektroden befinden. $K$. Pietrusky. [A. 101.]

\section{Über einen qualitativen Nachweis des Zirkoniums}

von IV ililela Biltz und Werner Mecklenburg. (Fingr.g. 20.77. 1912.)

In der älteren Literatur findet sich zum Nachweis des Zirkoniums in saurer Lösung Wasserstoffsuperoxyd vorgeschlagen ${ }^{1}$ ). Eine Nachprüfung dieses

I) B a il cy, Liehigs Ann. 232, 352 (1886).
Verfahrens führte schon vor längerer Zeit den einen der Vf. dieser Notiz zu der Erkênntnis, daß die Fällbarkeit des Zirkoniums durch dieses Reagens nicht dem Wasserstoffsuperoxyd als solchem, sondern der den älteren Handelspräparaten von Wasserstoffsuperoxyd meist beigemengten Phosphorsäure eigentümlich sei. Wie gelegentlich gesprächsweise konstatiert wurde, deckte sich diese Erfahrung mit der von $\mathrm{H}$ il l e b r a nd, der zur Bestimmung geringer Mengen Zirkoniums in Gesteinen die Natriumphosphatfällung in squrer Lösung benutzt ${ }^{2}$ ). Ater merkwürdigerweise scheint diese $\mathrm{H}$ ill le b r a $\mathrm{nd}$ sche Methode der Phosphatfällung für andere, z. B. qualitative $Z$ wecke wenig bekannt und benutzt zu sein. obgleich sie in ganz spezifischer Weise eine schnelle und eindeutige Ermittlung kleiner Zirkonmengen gestattet ${ }^{3}$ ). Auch in dem soeben erschienenen, ausgezeichneten Werke von R. J. M e y e $r$ und (). $\mathrm{H}$ a $\mathrm{u} \mathrm{s}$ e ${ }^{4}$ ) findet man sie in diesem Sinne nicht zitiert.

Säuert man eine zirkoniumhaltige Lösung mit Salpetersäure oder Salzsäure stark an, fügt einige Tropfen Natriumphosphatlösung hinzu und erwärmt, so fällt ein weißes Hydrogel, das Zirkon und Phosphorsäure enthält. Uber die Empfindlichkeit der Probe geben folgende Versuche einen Anhalt:

$5 \mathrm{ccm}$ der Lösung wurden mit $5 \mathrm{ccm}$ konz. Salzsäure oder $5 \mathrm{ccm}$ konz. Salpetersäure zum Sieden erhitzt und mit $2 \mathrm{ccm}$ Natriumphosphatlösung $(0,4$ molar. $)$ versetzt und nochmals aufgekocht

\section{Molarität von $\mathrm{ZrO}_{2}$ 0,00050 deutlicher Niederschlag, 0,00025 Reaktion unsicher.}

Ohne Säurezusatz hörte die Nachweisbarkeit schon bei 0,002 molar auf. Anwesenheit von Schwefelsäure verlangsamt die Reaktion sehr wesentlich.

Uns ist kein anderes an dieser Stelle der Analyse in Frage kommendes Element bekannt, das in s t a $r$ s a u r e r Lösung unter diesen Umständen einen Niederschlag gäbe. Eisen, Aluminium, die seltenen Erden, Thorium, Beryllium, Titan und Kieselsäure sind unwirksam.

Sehr bequem läßt sich der Nachweis im Maß3stabe der Lötrohrprobierkunst führen: Man schmilzt die auf Zirkonium zu prüfende Substanz in einer an einer Platindrahtspirale gehaltenen Sodaperle mit der oxydierenden Lötrohrflamme bei möglichst

2) W. F. H ill e b r and, Analyse der Silicatund Carbonatgesteine. Deutsche Ausgabe von E r n s tW i l k e - D ö r f u r t . Leipzig 1910, S. 144. ,Meine eigenen Versuche und diejenigen anderer, mit Wasserstoffsuperoxyd allein das Zirkon aus der Lösung seiner Sulfate niederzuschlagen, waren erfolglos bis auf Fälle, wo in sehr starken Lösungen und mit $30 \%$ igem Wasserstoffsuperoxyd gearbeitet wurde."

$\left.{ }^{3}\right)$ Arthur A. Noyes, William ('..Bray und Ellwood B. Spear (J. Am. Chem. Soc. 30, 516 [1908]) empfehlen den Nachwejs des Zirkons neben Titan mittels Phosphorsalzes in s $\mathrm{ch}$ wef el s a u rer Lösung. Nach unserer Frfahrung ist die Empfindlichkeit der Reaktion in salz- oder salpetersaurer Lösung wesentlich größer. (Anmerk. bei der Korrektur.)

4) R. J. Meyer u. O. Hauser. Die Analyse der seltenen Erden und der Erdsäuren, Stuttgart 1912 , S. 147 und $174-177$. 
hoher Temperatur ein, löst die gepulverte Perle in Salrsäure, kocht auf, filtriert und prüft die Lösung mit einem Tropfen Natriumphosphatlösung. Man ist somit in etwa 5 bis 10 Minuten über einen etwaigen wosentlichen Zirkongehalt der Probe unterrichtet. Bedingung ist natürlich, daß die Probe von vornherein nicht phuspliorsäurehaltig war. Enthielt sie Zinn, so ist es sicherer, dieses zunächst aus der Lösung mit Schwefelwanserstoffwasser zu entfernen, den Ammoniakniederschlag nach den $\mathbf{R e}$ geln der qualitativen Analyse zu bereiten und dessen salzsaure oder salpetersaure Lösung auf Zirkonium zu priiten.

Was die Natur der Zirkonerde-Phosphorsäurenied(rschläge anhetrifft, so int genaueres darïher noch nicht ermittelt worden; es liegt aber wohl nahe, sie als Analoga der Zinnsiture-Phosphorsüureniederschlize aufzufassen, die als Adsorptionsverbindungen der lieiden Komponenten erwiesen sind ${ }^{5}$ ). Fin Ersat\% der Zinnsäure durch saure Zirkonlösung bei der Phosphorsüureabscheidung in (iange der Analyse ist, wie lersuche ergeben halen, möglich; nur muB man sich damit hefreunden, day überschüssige Zirkonium nachtrüglich zu entfernen. oxler bei der Trennung seine Anwesenheit nelen dem Fisen mit in Kauf zil nehmen.

[A. 164.]

('lawathal i. H.. Chem. Labor. der Bergakademie.

\section{Waschen und Absorbieren von Oasen mit Hilfe von Flüssigkeitsstrahlen.}

Vorlüufige Mitteilung.

\section{Von Dr. Oskar Nagel, Wien.}

(Eingeg. 20.,6. 1912.)

Was Gegenstromprinzip beherrscht heute fast alle Vorgänge, in welchen (iase mit Flüssigkeiten gewaschen oder durch dienellen absorbiert werden, sowie diejenigen, in welchen tine Reaktion zwischen Gasen und Flüssigkeiten erzielt werden soll. Bis vor kurzem war diese Bevorzugung des Gegenwtrom. prinzips wohl herechtigt, weil es bei den bisherigen technischen Hilfsmitteln dem (ileichstromprinzip in jeder Hinwicht überlegen war. Durch die Vervollkommnung der Strahlapparate aher halien sich diese Beziehungen einigerma $B^{2} n$ verschohen, so da $B$ das Gleichstromprinzip infolge der durch die Strahlapparale gebotenen Vorteile. wie Handlichkeit, Raumersparnis usw. wieder \%u Eilıren kommen wird.

Die Urtype des Flizsigkeitsst rahlapparates, die llasserstralilpumpe. int allgemein trekannt. Sie ist in fast jeden Laboratorium zu finden und wird dasellost als Vakuumpumpe verwendet, also zur Bewegung von (iawen. Die Saugwirkung dieses Apparates wird dadurch hervorgerufen, dab ein Wasserstrahl mit großer (Geschwindigkeit aus einer engeren in eine weitere Diise übertritt. Was die Intensität dieser Saugwirkung, also den Wirkungsgrad dieses Apparates als Gasbewegungsmaschine anlangt, so ist dieselbe wehr gering, indem der weit größere Teil der im bewegten Strahle onthaltenen Energic in und zu der Bildung eines Wirbels, der sich in und besonders zwischen den Dïsen abspielt, verbraucht wird.

5) Werner Mecklanburg, Z. anorg. Chem. T4, 215 bis 221 (191:2).
Will man daher einen Strahlapparat zweckmäßig, also mit möglichst großem Nutzeffekte, verwenden, so muß vor allem dieser Wirbel also die intensive Vermischung der im Apparato zusammentretenden Medien ausgenutzt werden. Mit anderen Worten, die Flüssigkeitsatrahlapparate sind in erster Linie Mischmaschinen und erst in zweiter Linie Transportmaschinen. Und diese Flüssigkeitsstrahlapparate sind $e s$, welche vorteilhafterweise die Einführung des (ileichstromprinzipes gestatten, zumal da in den letzten Jahren die Strahlapparate sowohi in bezug auf Konstruktion als auch in bezug auf Iei-

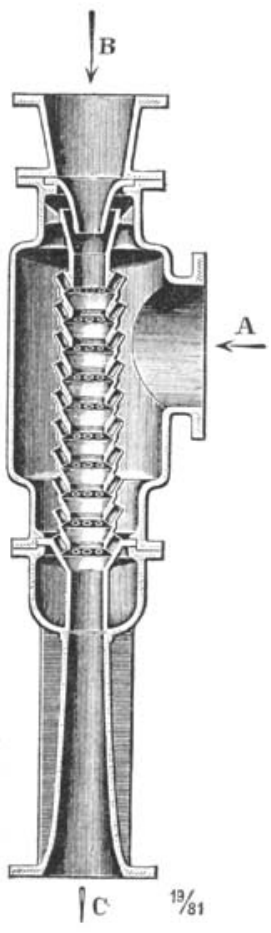

Fi:.. 1. stungsfähigkeit hedeutende Verbesserungen erfahren haben.

Winer dieser modernen, wirkungsvollen Strahlapparate, den ich hier als Beinpiel anführen will, ist der $\mathrm{K}$ o e r t in g sche Wasserstralilkon-

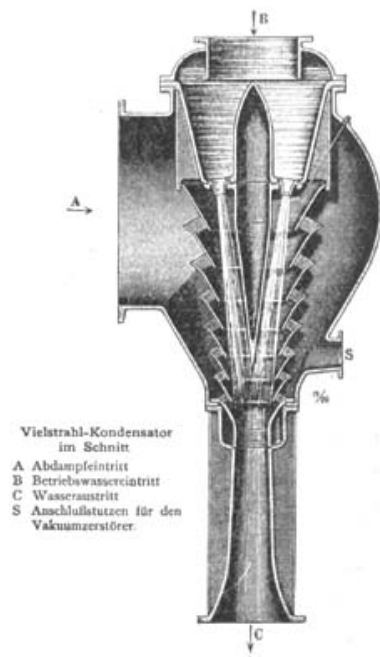

Fig. 2.

densator, der sowohl als einstrahliger als uuch als vielstrahliger Apparat gebaut wird. Die vielstrahlige Type ist infolge der gróßeren () herfläche des von ihr erzeugten sitrahles die wirksamert. Bisher sind diese Apparate ausschlieQlich als Kondensatoren in Dampfkraftanlagen verwendet worden, indem der Abdampf in den wirksamen Strahl hineingezogen und dadurch zugleich ein Vakuum erzeugt wurde. Ebendiese Maschinen können als (iasabsorptionsapparate verwendet werden, wobei an Stelle des Wesserstrahles irgendein Fiussigkeitsutrahl und an Stelle des Danupfes das zu alssorbierende (ias verwendet wird.

Im einfuchen Strahlenkondensator, Fig. I, läuft das Wasser, welches vortcilhafterweise einen Druck von ca. 4 Atm, haben soll - zur Beförderung des Wassers werden vorteilhafterweise Zent rifugalpumpen verwendet - vertikal von oben nach unten in Form eines zentralen strahles durch den Apparat, während der Abdampf durch die seitliche Óffnung eingesogen wird und durch die schief in das Strahl. rohr eingeschnittenen Offnungen zum wirksamen Wasserstrahl tritt. Der Wasserverbrauch dieser Tyde.pen. Minmouraviin von 60-17000 Litern. 